\title{
Skew cyclic displacements and decompositions of inverse matrix for an innovative structure matrix
}

\author{
Xiaoyu Jiang ${ }^{\mathrm{a}}$, Kicheon Hong ${ }^{\mathrm{a}}$, Zunwei Fu ${ }^{\mathrm{b}, *}$ \\ ${ }^{a}$ Department of Information and Telecommunications Engineering, The University of Suwon, Wau-ri, Bongdam-eup, Hwaseong-si, \\ Gyeonggi-do, 445-743, Korea. \\ ${ }^{b}$ Department of Mathematics, The University of Suwon, Wau-ri, Bongdam-eup, Hwaseong-si, Gyeonggi-do, 445-743, Korea.
}

Communicated by J. Brzdek

\begin{abstract}
In this paper, we study mainly on a class of column upper-minus-lower (CUML) Toeplitz matrices without standard Toeplitz structure, which are "similar" to the Toeplitz matrices. Their $(-1,-1)$-cyclic displacements coincide with cyclic displacement of some standard Toeplitz matrices. We obtain the formula on representation for the inverses of CUML Toeplitz matrices in the form of sums of products of $(-1,1)$-circulants and $(1,-1)$-circulants factor by constructing the corresponding displacement of the matrices. In addition, based on the relation between CUML Toeplitz matrices and CUML Hankel matrices, the inverse formula of CUML Hankel matrices can also be obtained. (C)2017 All rights reserved.
\end{abstract}

Keywords: CUML Toeplitz matrix, CUML Hankel matrix, skew cyclic displacement, RSFPLR circulants, RFMLR circulants, decomposition, inverse.

2010 MSC: 15A09, 15B05.

\section{Introduction}

Matrices have important applications in pure and applied mathematics [3, 24, 29]. Many scholars study their properties as well as the representation of their inverse formula. For example, in [31], Potts and Steidl gave attention to preconditioners for ill-conditioned Toeplitz matrices. The authors studied the hyponormality and spectra on Toeplitz operators in [5-7, 11]. Artzi and Shalom [4] presented that three columns of the inverse of a scalar Toeplitz matrix, when properly chosen, are always enough to reconstruct it. And on this basis Labahn and Shalom showed that the invertibility of a Toeplitz can be determined through the solvability of two standard equations, and the inverse matrix is represented by two of its columns and the entries of the original Toeplitz matrix in [25]. In addition, $\mathrm{Ng}$ et al. [30] proposed a modification of G. Labahn-T. Shalom theorem with another (shorter) proof. Labahn and Shalom [26] presented that formula for the inverse of layered or striped Toeplitz matrices are in the terms of solutions of standard equations. In [10], the inverse of an invertible Toeplitz matrix was obtained in the

\footnotetext{
${ }^{*}$ Corresponding author

Email addresses: jxy19890422@sina.com (Xiaoyu Jiang), Kchong@suwon.ac.kr (Kicheon Hong), zwfu@mail.bnu.edu.cn (Zunwei Fu)

doi:10.22436/jnsa.010.08.02
}

Received 2017-02-20 
form of Toeplitz Bezoutian of two columns. The Toeplitz inversion formula involving circulant matrices have also been proposed in $[2,27,28]$.

In [18], Jiang and Hong suggested the inverse formula of CUPL-Toeplitz matrices. Zheng et al. [34] gave the decomposition formulas of a class of CUPL Toeplitz matrices. The explicit inverse of nonsingular conjugate-Toeplitz and conjugate-Hankel matrices are provided in [12, 20]. Jiang and Wang [21] presented that the group inverse of an RFPL-Toeplitz matrix can be represented as the sum of products of lower and upper triangular Toeplitz matrices. The inverses of CUPL-Toeplitz and CUPL-Hankel matrices can be expressed by the Gohberg-Heinig type formula in [13].

It is well-known, in [23] that any matrix $A \in \mathbf{C}^{\mathfrak{n} \times \mathfrak{n}}$ is uniquely determined by its displacement, i.e., $\nabla_{0}(A)=A-Z_{0} A Z_{0}^{\top}$, where $Z_{0}$ is the lower shift matrix. Furthermore, Gohberg and Olshevsky [9] provided the new formula for representation of matrices (in particular, the Toeplitz matrices) and their inverses in the form of sums of products of factor circulants based on the analysis of the factor $\varphi$-cyclic displacement of matrices. Here the $\varphi$-cyclic displacement of a matrix $A \in \mathbf{C}^{\mathfrak{n} \times n}$ is defined as

$$
\nabla_{\varphi}(A)=A-Z_{\varphi} A Z_{\frac{1}{\varphi}}^{\top}
$$

where

$$
\mathrm{Z}_{\varphi}=\left(\begin{array}{ccccc}
0 & \cdots & \cdots & 0 & \varphi \\
1 & \ddots & & & 0 \\
0 & 1 & \ddots & & \vdots \\
\vdots & \ddots & \ddots & \ddots & \vdots \\
0 & \cdots & 0 & 1 & 0
\end{array}\right), \varphi \neq 0
$$

is the $\varphi$-cyclic lower shift matrix [9] (see also [1, 8] for case $\varphi=1$ ).

We derive formula for representation of the inverses of the column upper-minus-lower Toeplitz matrices and the column upper-minus-lower Hankel matrices based on the construct of new cyclic displacements of matrices in a more general situation (see (2.3) below for definition). This formula is involving with the factor $(-1,-1)$-circulants instead of the factor $\varphi$-circulants of the Toeplitz matrices that are the implications of the corresponding formula given in [9], and are useful for the analysis of the complexity of the inversion.

\section{Preliminaries}

According to the characteristics and applications of Toeplitz matrices and the matrices "close" to the Toeplitz matrices, in this paper we start to study a class of new type matrices "close" to Toeplitz matrices. More precisely, we deal with a column upper-minus-lower (CUML) Toeplitz matrix of the form as

$$
\mathrm{T}_{\mathrm{CUML}}=\left(\begin{array}{ccccc}
\mathrm{t}_{0} & \mathrm{t}_{-1} & \cdots & \mathrm{t}_{2-\mathrm{n}} & \mathrm{t}_{1-n} \\
\mathrm{t}_{1} & \mathrm{t}_{0}-\mathrm{t}_{1} & \ddots & \ddots & \mathrm{t}_{2-\mathrm{n}} \\
\mathrm{t}_{2} & \mathrm{t}_{1}-\mathrm{t}_{2} & \ddots & \ddots & \vdots \\
\vdots & \vdots & \ddots & \ddots & \mathrm{t}_{-1} \\
\mathrm{t}_{\mathrm{n}-1} & \mathrm{t}_{\mathrm{n}-2}-\mathrm{t}_{\mathrm{n}-1} & \cdots & \mathrm{t}_{1}-\mathrm{t}_{2} & \mathrm{t}_{0}-\mathrm{t}_{1}
\end{array}\right)_{\mathrm{n} \times \mathrm{n}}
$$

where $t_{0}, t_{ \pm 1}, \cdots, t_{ \pm(n-1)}$ are complex numbers.

It is clear, the entries $t_{i j}$ of the matrix in (2.1) are given by the following formula:

$$
t_{i j}= \begin{cases}t_{i-j,} & j=1 \text { or } j>i, \\ t_{i-j}-t_{i-j+1}, & 2 \leqslant j \leqslant i .\end{cases}
$$


In particular, if $t_{1-n}=t_{1}, t_{2-n}=t_{2}, \cdots, t_{-1}=t_{n-1}$, then $T_{C U M L}$ is a row first-minus-last right circulant matrix, which is firstly defined in [32].

In passing, a column upper-minus-lower (CUML) Hankel matrix is of the form as

$$
\mathrm{H}_{\mathrm{CUML}}=\left(\begin{array}{ccccc}
h_{0} & h_{1} & \cdots & h_{n-2} & h_{n-1} \\
h_{1} & . \cdot & . & h_{n-1}-h_{n} & h_{n} \\
\vdots & . \cdot & . & h_{n}-h_{n+1} & h_{n+1} \\
h_{n-2} & . \cdot & . & \vdots & \vdots \\
h_{n-1}-h_{n} & h_{n}-h_{n+1} & \cdots & h_{2 n-3}-h_{2 n-2} & h_{2 n-2}
\end{array}\right)_{n \times n},
$$

where $h_{0}, h_{1}, \cdots, h_{2 n-2}$ are complex numbers.

Obviously, the entries $h_{i j}$ of the matrix in (2.2) are given by the following formula:

$$
h_{i j}= \begin{cases}h_{i+j-2,} & j=n \text { or } i+j \leqslant n, \\ h_{i+j-2}-h_{i+j-1}, & i+j>n \text { and } j<n .\end{cases}
$$

Specially, if $h_{0}=h_{n}, h_{1}=h_{n+1}, \cdots, h_{n-2}=h_{2 n-2}$, then $H_{\text {CUML }}$ is called a row last-minus-first leftcirculant matrix, which is firstly defined in [32].

It should be noted that $\mathrm{H}_{\mathrm{CUML}} \mathrm{J}$ is a CUML Toeplitz matrix, where $\mathrm{J}$ is the "reverse unit matrix", having ones along the antidiagonal and zeros elsewhere.

The $(-1,-1)$-cyclic displacement of a matrix $A \in \mathbf{C}^{\mathrm{n} \times \mathrm{n}}$ is defined as

$$
\nabla_{-1,-1}(A)=A-\Xi_{-1,-1} A \Xi_{-1,-1}^{-1}
$$

where

$$
\Xi_{-1,-1}=\left(\begin{array}{cccccc}
0 & 0 & \cdots & \cdots & 0 & -1 \\
1 & -1 & \ddots & & & 0 \\
0 & 1 & -1 & \ddots & & \vdots \\
\vdots & \ddots & \ddots & \ddots & \ddots & \vdots \\
\vdots & & \ddots & 1 & -1 & 0 \\
0 & \cdots & \cdots & 0 & 1 & -1
\end{array}\right), \quad \Xi_{-1,-1}^{-1}=\left(\begin{array}{cccccc}
-1 & 1 & 1 & 1 & \cdots & 1 \\
-1 & 0 & 1 & \ddots & \ddots & \vdots \\
\vdots & \vdots & \ddots & \ddots & \ddots & 1 \\
-1 & \vdots & & \ddots & \ddots & 1 \\
-1 & \vdots & & & \ddots & 1 \\
-1 & 0 & \cdots & \cdots & \cdots & 0
\end{array}\right),
$$

$\Xi_{-1,-1}$ is the $(-1,-1)$-cyclic lower shift matrix. The integer number $\epsilon=\operatorname{rank} \nabla_{-1,-1}(A)$ is referred to as $(-1,-1)$-cyclic displacement rank of the matrix $A$. Matrix $A$ has $(-1,-1)$-cyclic displacement structure (with respect to $\Xi_{-1,-1}$ ), if $\epsilon$ is comparatively small.

According to the linear transformation $\nabla_{-1,-1}(\cdot)$ in $\mathbf{C}^{\mathfrak{n} \times \mathfrak{n}}$ presented via (2.3), it is quite clear that for an invertible matrix $A \in \mathbf{C}^{\mathfrak{n} \times \mathfrak{n}}$, a relation exists between the $(-1,-1)$-cyclic displacements of the inverse matrix $A^{-1}$ and the $(-1,-1)$-cyclic displacement of $A$, namely

$$
\nabla_{-1,-1}(A)=-A \cdot \nabla_{-1,-1}\left(A^{-1}\right) \cdot \Xi_{-1,-1} A \Xi_{-1,-1}^{-1} \cdot
$$

From (2.4), we know that the $(-1,-1)$-cyclic displacement rank is inherited under matrix inversion $\operatorname{rank} \nabla_{-1,-1}(A)=\operatorname{rank} \nabla_{-1,-1}\left(A^{-1}\right)$. Utilizing the $(-1,-1)$-cyclic displacement technique, the equation (2.4) provides a way of constructing the $(-1,-1)$-cyclic displacement of the inverse matrix of A. If, in particular, the $(-1,-1)$-cyclic displacement of $A \in \mathbf{C}^{\mathfrak{n} \times \mathfrak{n}}$ is given as the outer sum

$$
\nabla_{-1,-1}(A)=\sum_{i=1}^{\epsilon} \mathbf{a}_{i} \cdot \mathbf{b}_{i}^{\top},
$$

where $\mathbf{a}_{i}, \mathbf{b}_{i} \in \mathbf{C}^{\mathfrak{n}}, \mathfrak{i}=1,2, \cdots, \epsilon, \epsilon=\operatorname{rank} \nabla_{-1,-1}(A)$, from (2.4), the analogous representation for 
$\nabla_{-1,-1}\left(A^{-1}\right)$ can be received by solving $2 \epsilon$ matrix equations, involving the matrix $A$ and the vectors of outer sum (2.5):

$$
\nabla_{-1,-1}\left(A^{-1}\right)=-\sum_{i=1}^{\epsilon}\left(A^{-1} \mathbf{a}_{i}\right) \cdot\left(\mathbf{b}_{i}^{\top} \Xi_{-1,-1} A^{-1} \Xi_{-1,-1}^{-1}\right) .
$$

According to the above statement, set $\hat{\mathbf{b}}_{i}^{\top}=\mathbf{b}_{i}^{\top} \cdot \Xi_{-1,-1}(i=1,2, \cdots, \epsilon)$ and let the vectors $\mathbf{f}_{i}$ and $\hat{\mathbf{s}}_{i}^{\top}$ be the solutions of the following equations

$$
A \mathbf{f}_{i}=\mathbf{a}_{i}(i=1,2, \cdots, \epsilon),
$$

and

$$
\hat{\mathbf{s}}_{i}^{\top} A=\hat{\mathbf{b}}_{i}^{\top}(i=1,2, \cdots, \epsilon),
$$

furthermore, in view of (2.4), we have

$$
\nabla_{-1,-1}\left(A^{-1}\right)=-\sum_{i=1}^{\epsilon} \mathbf{f}_{i} \cdot \mathbf{s}_{i}^{\top}
$$

where

$$
\mathbf{s}_{i}^{\top}=\hat{\mathbf{s}}_{i}^{\top} \cdot \Xi_{-1,-1}^{-1} \cdot
$$

Solving the equations

$$
\mathbf{y}_{1}^{\top} A=\mathbf{e}_{0}^{\top}
$$

with $\mathbf{e}_{0}^{\top}=(1,0, \cdots, 0) \in \mathbf{C}^{\mathfrak{n}}$, and

$$
A \mathbf{y}_{2}=\mathbf{e}_{0}
$$

yields the first row and the first column of $A^{-1}$, respectively. Note that, in our consideration, the matrix $A$ is supposed to be invertible from the very beginning.

Moreover, the solvability of equations (2.6) and (2.11) implies invertibility of $A$. Indeed, let $\mathbf{f}_{i}(i=$ $1,2, \cdots, \epsilon)$ and $\mathbf{y}_{2}$ be the solutions of (2.6) and (2.11), respectively, and let $\mathbf{p}^{\top} A=0$ with $\mathbf{p}=\left(p_{0}, p_{1}, \cdots\right.$, $\left.p_{n-1}\right)^{\top} \in \mathbf{C}^{n}$. Then

$$
\mathbf{p}^{\top}\left(A-\Xi_{-1,-1} A \Xi_{-1,-1}^{-1}\right) \Xi_{-1,-1} \stackrel{(2.5)}{=} \mathbf{p}^{\top} \sum_{i=1}^{\epsilon} \mathbf{a}_{i} \cdot \mathbf{b}_{i}^{\top} \Xi_{-1,-1} \stackrel{(2.6)}{=} \mathbf{p}^{\top} A \sum_{i=1}^{\epsilon} \mathbf{f}_{i} \cdot \mathbf{b}_{i}^{\top} \Xi_{-1,-1}=0,
$$

so that $\mathbf{p}^{\top} \Xi_{-1,-1} A=0$. From

$$
\mathbf{p}^{\top} \Xi_{-1,-1}\left(A-\Xi_{-1,-1} A \Xi_{-1,-1}^{-1}\right) \Xi_{-1,-1}=\mathbf{p}^{\top} \Xi_{-1,-1} A \sum_{i=1}^{\epsilon} \mathbf{f}_{i} \mathbf{b}_{i}^{\top} \Xi_{-1,-1}=0,
$$

it follows that $\mathbf{p}^{\top} \Xi_{-1,-1}^{2} A=0$. In this way, we get finally

$$
\mathbf{p}^{\top} \Xi_{-1,-1}^{k} A=0, k=0,1, \cdots, \mathfrak{n}-1 .
$$

Post-multiplying the last identities by the solution $\mathbf{y}_{2}$ of the equation (2.11), we can get the following formula

$$
0=\mathbf{p}^{\top} \Xi_{-1,-1}^{k} A \mathbf{y}_{2}=\mathbf{p}^{\top} \Xi_{-1,-1}^{k} \mathbf{e}_{0}, \mathrm{k}=0,1, \cdots, \mathrm{n}-1,
$$

i.e., 


$$
\begin{aligned}
\mathbf{p}_{0} & =\mathbf{p}^{\top} \mathbf{e}_{0}=0, \mathbf{p}_{1}=\mathbf{p}^{\top} \Xi_{-1,-1} \mathbf{e}_{0}=\mathbf{p}^{\top} \mathbf{e}_{1}=0, \\
\mathbf{p}_{\mathrm{k}} & =\mathbf{p}^{\top} \Xi_{-1,-1}^{k} \mathbf{e}_{0} \\
& =\mathbf{p}^{\top}\left[0,(-1)^{\mathrm{k}-1}, \mathrm{C}_{\mathrm{k}-1}^{1}(-1)^{\mathrm{k}-2}, \mathrm{C}_{\mathrm{k}-1}^{2}(-1)^{\mathrm{k}-3}, \cdots,-\mathrm{C}_{\mathrm{k}-1}^{\mathrm{k}-2}, 1,0, \cdots, 0\right]^{\top}=0, \mathrm{k}=2,3, \cdots, \mathrm{n}-1,
\end{aligned}
$$

where $C_{n}^{i}$ is binomial coefficient $\left(\begin{array}{c}n \\ i\end{array}\right)$. Given the above, we can conclude all coordinates of the vector $\mathbf{p}$ are equal to zero, and hence $A$ is invertible. Analogously, we may show that the solvability of equations (2.7) and (2.10) yields the invertibility of $A$ as well.

Thus, we have proved the following.

Theorem 2.1. Let $A \in \mathbf{C}^{n \times n}$, and $\nabla_{-1,-1}(A)$ is given by (2.5). If the equations (2.6) and (2.11) ((2.7) and (2.10), respectively) have the solutions $\mathbf{f}_{i}$ and $\mathbf{y}_{2}\left(\hat{\mathbf{s}}_{i}^{\top}\right.$ and $\left.\mathbf{y}_{1}^{\top}\right)$, respectively, then $A$ is invertible, and thus (2.7) and (2.10) ((2.6) and (2.11), respectively) are solvable, and $\nabla_{-1,-1}\left(A^{-1}\right)$ is of the form as in (2.8) with $\mathbf{s}_{i}^{\top}=\hat{\mathbf{s}}_{i}^{\top} \cdot \Xi_{-1,-1}^{-1}, \mathbf{i}=$ $1,2, \cdots, \epsilon$.

\section{Factor circulant decompositions of CUML Toeplitz matrices}

Before stating the main results, we first introduce the related notations. In [14-16], RSFPLRcircfr $\left(\mathbf{p}^{\top}\right)$ denotes the row skew first-plus-last right circulant matrix with the first row $\mathbf{p}^{\top}=\left[\begin{array}{lllll}p_{0} & p_{1} & \cdots & p_{n-1}\end{array}\right]$, i.e., the matrix of the form

$$
\operatorname{RSFPLRcircfr}\left(\mathbf{p}^{\top}\right)=\left(\begin{array}{ccccc}
p_{0} & p_{1} & p_{2} & \cdots & p_{n-1} \\
-p_{n-1} & p_{0}+p_{n-1} & \ddots & \ddots & \vdots \\
\vdots & -p_{n-1}+p_{n-2} & \ddots & \ddots & p_{2} \\
-p_{2} & \vdots & \ddots & \ddots & p_{1} \\
-p_{1} & -p_{2}+p_{1} & \cdots & -p_{n-1}+p_{n-2} & p_{0}+p_{n-1}
\end{array}\right)
$$

It can be seen that it is a matrix with an arbitrary first row and the following rule for obtaining any other row from the previous one: get the $i+1$ st row by adding the last element of the ith row to the first element of the ith row, and -1 times the last element of the ith row, and then shifting the elements of the ith row (cyclically) one position to the right.

In this paper, we denote the row skew first-plus-last right circulant with the first column $\mathbf{p}=\left[\begin{array}{llll}p_{0} & p_{1} & \cdots\end{array}\right.$ $\left.p_{n-1}\right]^{\top}$ by RSFPLRcircfc $(\mathbf{p})$, i.e., the matrix of the form

$$
\operatorname{RSFPLRcircfc}(\mathbf{p})=\left(\begin{array}{ccccc}
p_{0} & -p_{n-1} & -p_{n-2} & \cdots & -p_{1} \\
p_{1} & p_{0}-p_{1} & \ddots & \ddots & \vdots \\
p_{2} & p_{1}-p_{2} & \ddots & \ddots & -p_{n-2} \\
\vdots & \vdots & \ddots & \ddots & -p_{n-1} \\
p_{n-1} & p_{n-2}-p_{n-1} & \cdots & p_{1}-p_{2} & p_{0}-p_{1}
\end{array}\right) .
$$

If need be, we shall refer to such matrices $\operatorname{RSFPLR\operatorname {circfr}}\left(\mathbf{p}^{\top}\right)$ and $\operatorname{RSFPLRcircfc}(\mathbf{p})$ as factor $(-1,1)$-circulants. It should be noted that if $\mathbf{p}^{\top}=\left(p_{0}, p_{1}, \cdots, p_{n-1}\right)$, then

$$
\operatorname{RSFPLR} \operatorname{circfr}\left(\mathbf{p}^{\top}\right)=\operatorname{RSFPLR} \operatorname{circfc}(\tilde{\mathbf{p}})
$$

with $\tilde{\mathbf{p}}=\left(\begin{array}{lllll}p_{0} & -p_{n-1} & -p_{n-2} & \cdots & -p_{1}\end{array}\right)^{\top}$, and that the identity

$$
\operatorname{RSFPLRcircfr}\left(\mathbf{p}^{\top}\right) \operatorname{RSFPLR} \operatorname{circfr}\left(\mathbf{q}^{\top}\right)=\operatorname{RSFPLR} \operatorname{circfr}\left(\mathbf{q}^{\top}\right) \operatorname{RSFPLRcircfr}\left(\mathbf{p}^{\top}\right)
$$

and

$$
\operatorname{RSFPLRcircfc}(\mathbf{p}) \operatorname{RSFPLRcircfc}(\mathbf{q})=\operatorname{RSFPLR} \operatorname{circfc}(\mathbf{q}) \operatorname{RSFPLRcircfc}(\mathbf{p})
$$

holds for any column vector $\mathbf{p}, \mathbf{q} \in \mathbf{C}^{\mathfrak{n}}$. 
In [32], RFMLRcircfr $\left(\mathbf{p}^{\top}\right)$ denotes the row first-minus-last right circulant matrix with the first row $\mathbf{p}^{\top}=\left[\begin{array}{llll}p_{0} & p_{1} & \cdots & p_{n-1}\end{array}\right]$, i.e., the matrix of the form

$$
\operatorname{RFMLRcircfr}\left(\mathbf{p}^{\top}\right)=\left(\begin{array}{ccccc}
p_{0} & p_{1} & p_{2} & \cdots & p_{n-1} \\
p_{n-1} & p_{0}-p_{n-1} & \ddots & \ddots & \vdots \\
\vdots & p_{n-1}-p_{n-2} & \ddots & \ddots & p_{2} \\
p_{2} & \vdots & \ddots & \ddots & p_{1} \\
p_{1} & p_{2}-p_{1} & \cdots & p_{n-1}-p_{n-2} & p_{0}-p_{n-1}
\end{array}\right) .
$$

The matrix over $\mathrm{C}$ has an arbitrary first row; the following rule is applied to obtain any other row from the previous one: get the $i+1$ st row by subtracting the last element of the $i$ th row from the first element of the ith row, and then shifting the elements of the ith row (cyclically) one position to the right. In this paper, we describe the row first-minus-last right circulant with the first column $\mathbf{p}=\left[\begin{array}{lllll}p_{0} & p_{1} & \cdots & p_{n-1}\end{array}\right]^{\top}$ by RFMLRcircfc(p), i.e., the matrix of the form

$$
\operatorname{RFMLRcircfc}(\mathbf{p})=\left(\begin{array}{ccccc}
p_{0} & p_{n-1} & p_{n-2} & \cdots & p_{1} \\
p_{1} & p_{0}-p_{1} & \ddots & \ddots & \vdots \\
p_{2} & p_{1}-p_{2} & \ddots & \ddots & p_{n-2} \\
\vdots & \vdots & \ddots & \ddots & p_{n-1} \\
p_{n-1} & p_{n-2}-p_{n-1} & \cdots & p_{1}-p_{2} & p_{0}-p_{1}
\end{array}\right) .
$$

If need be, we shall refer such matrices $\operatorname{RFMLR\operatorname {circfr}}\left(\mathbf{p}^{\top}\right)$ and $\operatorname{RFMLR\operatorname {Rirff}}(\mathbf{p})$ as factor $(1,-1)$ circulants. It should be noted that if $\mathbf{p}^{\top}=\left(p_{0}, p_{1}, \cdots, p_{n-1}\right)$, then

$$
\operatorname{RFMLR\operatorname {circfr}}\left(\mathbf{p}^{\top}\right)=\operatorname{RFMLR\operatorname {Rircfc}}(\tilde{\mathbf{p}})
$$

with $\tilde{\mathbf{p}}=\left(\mathrm{p}_{0}, \mathrm{p}_{\mathrm{n}-1}, \mathrm{p}_{\mathrm{n}-2}, \cdots, \mathrm{p}_{1}\right)^{\mathrm{T}}$, and that the identity

$$
\operatorname{RFMLR\operatorname {ircfr}}\left(\mathbf{p}^{\top}\right) \operatorname{RFMLR\operatorname {circfr}}\left(\mathbf{q}^{\top}\right)=\operatorname{RFMLR\operatorname {circfr}}\left(\mathbf{q}^{\top}\right) \operatorname{RFMLR\operatorname {circfr}}\left(\mathbf{p}^{\top}\right)
$$

and

$$
\operatorname{RFMLR\operatorname {Rirfc}}(\mathbf{p}) \operatorname{RFMLR\operatorname {Rirfc}}(\mathbf{q})=\operatorname{RFMLR\operatorname {Rirfc}}(\mathbf{q}) \operatorname{RFMLR\operatorname {Rircfc}}(\mathbf{p})
$$

holds for any column vector $\mathbf{p}, \mathbf{q} \in \mathbf{C}^{\mathrm{n}}$.

In particular, let $T_{\text {CUML }}$ be an $n \times n$ CUML Toeplitz matrix with $\left(t_{0}, t_{-1}, \cdots, t_{1-n}\right)$ and $\left(t_{0}, t_{1}, \cdots, t_{n-1}\right)^{\top}$ as its first row and first column, respectively. Considering the $(-1,-1)$-cyclic displacement of $T_{C U M L}$, we have

$$
\begin{aligned}
\nabla_{-1,-1}\left(T_{\text {CUML }}\right) & =T_{\text {CUML }}-\Xi_{-1,-1} T_{\text {CUML }} \Xi_{-1,-1}^{-1} \\
& =\left(\begin{array}{cccc}
0 & t_{-1}+t_{n-1} & \cdots & t_{1-n}+t_{1} \\
t_{1}+t_{1-n} & 0 & \cdots & 0 \\
\vdots & \vdots & & \vdots \\
t_{n-1}+t_{-1} & 0 & \cdots & 0
\end{array}\right) \\
& =\left(\begin{array}{c}
\beta \\
t_{1}+t_{1-n} \\
\vdots \\
t_{n-1}+t_{-1}
\end{array}\right) \cdot \mathbf{e}_{0}^{\top}+\mathbf{e}_{0} \cdot\left(\begin{array}{llll}
-\beta & t_{-1}+t_{n-1} & \cdots & t_{1-n}+t_{1}
\end{array}\right) \\
& =\mathbf{x} \cdot \mathbf{e}_{0}^{\top}+\mathbf{e}_{0} \cdot \mathbf{z}^{\top},
\end{aligned}
$$


where $\beta$ may be an arbitrary complex number, $\mathbf{x}=\left(\beta, t_{1}+t_{1-n}, \cdots, t_{n-1}+t_{-1}\right)^{\top}, \mathbf{z}^{\top}=\left(-\beta, t_{-1}+\right.$ $\left.t_{n-1}, \cdots, t_{1-n}+t_{1}\right), \mathbf{e}_{0}=(1,0, \cdots, 0)^{\top} \in \mathbf{C}^{n}$.

Obviously, $(-1,-1)$-cyclic displacement rank for the CUML Toeplitz matrix is less than or equal to 2, so that such $\mathrm{T}_{\mathrm{CUML}}$ has $(-1,-1)$-cyclic displacement structure if $\mathrm{n}$ is sufficiently large.

Furthermore, in the CUML Toeplitz matrix case, $\nabla_{-1,-1}\left(\mathrm{~T}_{\mathrm{CUML}}\right)$ also has the specific form given by (3.1). Then the equations (2.6) and (2.7) reduce respectively to

$$
\mathrm{T}_{\mathrm{CUML}} \mathbf{f}_{1}=\mathbf{x} \text { and } \mathrm{T}_{\mathrm{CUML}} \mathbf{f}_{2}=\mathbf{e}_{0},
$$

and

$$
\hat{\mathbf{s}}_{1}^{\top} \mathrm{T}_{\mathrm{CUML}}=\mathbf{e}_{0}^{\top} \Xi_{-1,-1} \text { and } \hat{\mathbf{s}}_{2}^{\top} \mathrm{T}_{\mathrm{CUML}}=\mathbf{z}^{\top} \Xi_{-1,-1} .
$$

Thus, by (2.8), we have

$$
\nabla_{-1,-1}\left(\mathrm{~T}_{\mathrm{CUML}}^{-1}\right)=-\sum_{i=1}^{2} \mathbf{f}_{i} \cdot \mathbf{s}_{i}^{\top}
$$

where

$$
\mathbf{f}_{1}=\mathrm{T}_{\mathrm{CUML}}^{-1} \mathbf{x}, \quad \mathbf{s}_{1}^{\top}=\mathbf{e}_{0}^{\top} \Xi_{-1,-1} \mathrm{~T}_{\mathrm{CUML}}^{-1} \Xi_{-1,-1}^{-1}, \quad \mathbf{f}_{2}=\mathrm{T}_{\mathrm{CUML}}^{-1} \mathbf{e}_{0}, \quad \mathbf{s}_{2}^{\top}=\mathbf{z}^{\top} \Xi_{-1,-1} \mathrm{~T}_{\mathrm{CUML}}^{-1} \Xi_{-1,-1}^{-1} .
$$

Then from (2.5) and [1,8], we can easily obtain the next theorem.

Theorem 3.1. If the equality

$$
\nabla_{-1,-1}\left(\mathrm{~T}_{\mathrm{CUML}}\right)=\sum_{i=1}^{\epsilon} \mathbf{a}_{i} \cdot \mathbf{b}_{i}^{\top}, \quad\left(\mathbf{a}_{i}, \mathbf{b}_{i} \in \mathbf{C}^{\mathfrak{n}}\right)
$$

holds, then

$$
\mathrm{T}_{\mathrm{CUML}}=\operatorname{RSFPLR} \operatorname{circfc}\left(\mathrm{T}_{\mathrm{CUML}}\right)+\sum_{i=1}^{\epsilon} \mathrm{L}\left(\mathbf{a}_{\mathrm{i}}\right) \cdot \operatorname{SCirc}\left(\mathbf{b}_{\mathrm{i}}^{\mathrm{T}}\right),
$$

where RSFPLRcircfC( $\left.\mathrm{T}_{\mathrm{CUML}}\right)$ is the row skew first-plus-last right circulant with the same first column as that of $\mathrm{T}_{\mathrm{CUML}}$, and $\mathrm{L}\left(\mathbf{a}_{\mathrm{i}}\right)$ is the lower triangular Toeplitz matrix with the first column $\mathbf{a}_{\mathrm{i}}=\left(\mathrm{a}_{\mathrm{i} 0}, \mathrm{a}_{\mathrm{i} 1}, \cdots, \mathrm{a}_{\mathrm{i}, \mathrm{n}-1}\right)^{\mathrm{T}}$, and $\operatorname{SCirc}\left(\mathbf{b}_{i}^{T}\right)$ is the skew circulant matrix with the first row $\mathbf{b}_{i}^{\top}=\left(b_{i 0}, b_{i 1}, \cdots, b_{i, n-1}\right),[17,19,22,33]$.

Theorem 3.2. Let $\nabla_{-1,-1}(\cdot)$ be the linear operator in $\mathbf{C}^{\mathrm{n} \times \mathrm{n}}$ defined by (2.3). Then the following statements hold:

(i) the equality $\nabla_{-1,-1}(A)=0$ holds if and only if $A$ is a row skew first-plus-last right circulant matrix;

(ii) if the equation

$$
\nabla_{-1,-1}(X)=\sum_{i=1}^{\epsilon} \mathbf{a}_{i} \cdot \mathbf{b}_{i}^{\top},
$$

where $\mathbf{a}_{i}, \mathbf{b}_{i}^{\top}(\mathfrak{i}=1,2, \cdots, \epsilon)$ are given vectors, is solvable with respect to $\mathbf{X} \in \mathbf{C}^{\mathfrak{n} \times n}$, then

$$
\sum_{i=1}^{\epsilon} \operatorname{RSFPLRcircfc}\left(\mathbf{a}_{i}\right) \cdot \operatorname{RSFPLRcircfr}\left(\mathbf{b}_{i}^{\top}\right)=0 ;
$$

(iii) if $2 \epsilon$ vectors $\mathbf{a}_{i}$ and $\mathbf{b}_{i}^{\top}(\boldsymbol{i}=1,2, \cdots, \epsilon)$ satisfy the condition (3.6), then the equation (3.5) has the solution

$$
X=\operatorname{RSFPLRcircfc}(X)+\frac{1}{2} \sum_{i=1}^{\epsilon} \operatorname{RFMLRcircfc}\left(\mathbf{a}_{i}\right) \cdot \operatorname{RSFPLRcircfr}\left(\mathbf{b}_{i}^{\top}\right),
$$

where RSFPLRcircf $(\mathrm{X})$ is the row skew first-plus-last right circulant with the same first column as that of $\mathrm{X}$; 
(iv) under the conditions of the assertion (iii) the solution $\mathrm{X}$ of the equation (3.5) may also be written in the form

$$
X=\operatorname{RSFPLRcircfr}(X)+\frac{1}{2} \sum_{i=1}^{\epsilon} \operatorname{RSFPLRcircfc}\left(\mathbf{a}_{i}\right) \cdot \operatorname{RFMLRcircfr}\left(\mathbf{b}_{i}^{\top}\right),
$$

where RSFPLRcircfr(X) is the row skew first-plus-last right circulant with the same first row as that of $\mathrm{X}$.

Proof.

(i) Let matrix $A=\left(a_{i j}\right)_{i, j=0}^{n-1}$ meet the requirement $\nabla_{-1,-1}(A)=0$, i.e., $A=\Xi_{-1,-1} A \Xi_{-1,-1}^{-1}$. From this equality, it follows that:

$$
\begin{array}{ll}
a_{i j}=a_{i+1, j+1}, & \text { if } j \neq 0, \\
a_{0 j}=-a_{n-j, 0}, & \text { if } j \neq 0, \\
a_{i 0}=-a_{0, n-i}, & \text { if } i \neq 0, \\
a_{i 1}=a_{i-1,0}+a_{i, 0}, & \text { if } i \neq 0 .
\end{array}
$$

By definition, these relations describe that $A$ is a row skew first-plus-last right circulant matrix.

On the other hand, if $A$ is a row skew first-plus-last right circulant matrix, calculating by the formula (2.3) we get $\nabla_{-1,-1}(\mathrm{~A})=0$.

(ii) Let $\nabla_{-1,-1}(X)=\sum_{i=1}^{\epsilon} \mathbf{a}_{i} \cdot \mathbf{b}_{i}^{\top}$. Then taking into account the equality (2.3), we have

$$
\begin{aligned}
0 & =\sum_{j=0}^{n-1} \Xi_{-1,-1}^{j} \cdot\left(X-\Xi_{-1,-1} X \Xi_{-1,-1}^{-1}\right) \cdot\left(\Xi_{-1,-1}^{\top}\right)^{j} \\
& =\sum_{j=0}^{n-1} \sum_{i=1}^{\epsilon}\left(\Xi_{-1,-1}^{j} \mathbf{a}_{i}\right) \cdot\left(\mathbf{b}_{i}^{\top}\left(\Xi_{-1,-1}^{\top}\right)^{j}\right)=\sum_{i=1}^{\epsilon} \operatorname{RSFPLRcircfc}\left(\mathbf{a}_{i}\right) \cdot \operatorname{RSFPLRcircfr}\left(\mathbf{b}_{i}^{\top}\right) .
\end{aligned}
$$

The last equality follows from the general identity $U \cdot V^{\top}=\sum_{k=0}^{n-1} \mathbf{f}_{k} \cdot \mathbf{s}_{k}^{\top}$, where $\mathbf{f}_{k}$ and $\mathbf{s}_{k}$ are the k-th columns of the matrices $\mathrm{U}$ and $\mathrm{V}$, correspondingly. The assertion (ii) is proved.

(iii) Suppose that vectors $\mathbf{a}_{i}, \mathbf{b}_{\mathfrak{i}}(\mathfrak{i}=1,2, \cdots, \epsilon)$ satisfy the condition (3.6) and compute the (-1,-1)-cyclic displacement of the matrix $X$ defined by (3.7). Therefore we have

$$
\nabla_{-1,-1}(X)=\nabla_{-1,-1}(\operatorname{RSFPLRcircfc}(X))+\frac{1}{2} \sum_{i=1}^{\epsilon} \nabla_{-1,-1}\left(\operatorname{RFMLRcircfc}\left(\mathbf{a}_{i}\right)\right) \cdot \operatorname{RSFPLRcircfr}\left(\mathbf{b}_{i}^{\top}\right) .
$$

Clearly, $(-1,-1)$-cyclic displacement for RFMLRcircfc(r) with the first column $\mathbf{r}=\left[\begin{array}{llll}r_{0} & r_{1} & \cdots & r_{n-1}\end{array}\right]^{\top}$, has the following simple form

$$
\nabla_{-1,-1}(\operatorname{RFMLRcircfc}(\mathbf{r}))=\left(\begin{array}{cccc}
0 & 2 r_{n-1} & \cdots & 2 r_{1} \\
2 r_{1} & 0 & \cdots & 0 \\
\vdots & \vdots & & \vdots \\
2 r_{n-1} & 0 & \cdots & 0
\end{array}\right)=2\left(\mathbf{r} \cdot \mathbf{e}_{0}^{\top}-\mathbf{e}_{0} \cdot \tilde{\mathbf{r}}^{\top}\right)
$$

where $\tilde{\mathbf{r}}^{\top}=\left[r_{0}-r_{n-1}-r_{n-2} \cdots-r_{1}\right]$ is the first row of the RSFPLRcircfc $(\mathbf{r})$. Calculating in this way, the $(-1,-1)$-cyclic displacement for each matrix RFMLRcircfc $\left(\mathbf{a}_{i}\right)$ on the right hand side of (3.8) and taking into account that $\nabla_{-1,-1}(\operatorname{RSFPLRcircfc}(X))=0$ in view of (i), we have

$$
\nabla_{-1,-1}(X)=\sum_{i=1}^{\epsilon} \mathbf{a}_{i} \cdot \mathbf{e}_{0}^{\top} \cdot \operatorname{RSFPLRcircfr}\left(\mathbf{b}_{i}^{\top}\right)-\sum_{i=1}^{\epsilon} \mathbf{e}_{0} \cdot \tilde{\mathbf{a}}_{i}^{\top} \cdot \operatorname{RSFPLRcircfr}\left(\mathbf{b}_{i}^{\top}\right),
$$


where $\tilde{\mathbf{a}}_{i}^{\top}$ are the first rows of the matrices RSFPLRcircfc $\left(\mathbf{a}_{i}\right)(i=1,2, \cdots, \epsilon)$. Therefore, in view of (3.6) the sum of the last $\epsilon$ terms in (3.9) is equal to zero matrix. Furthermore, $\mathbf{e}_{0}^{\top} \cdot \operatorname{RSFPLR} \operatorname{circfr}\left(\mathbf{b}_{i}^{\top}\right)=\mathbf{b}_{i}^{\top}$ $(i=1,2, \cdots, \epsilon)$, and hence the matrix $X$ defined by (3.7) satisfies the equation (3.5), therefore, in view of (3.6), the first columns of the matrices $X$ and RSFPLRcircfc $(X)$ coincide. The assertion (iii) is now completely proved.

(iv) The assertion (iv) can be proved with the same arguments.

According to the proposition (i) of Theorem 3.2, every complex matrix $A$ is determined by its $(-1,-1)$ cyclic displacement up to a row skew first-plus-last right circulant matrix. Therefore, an arbitrary complex matrix is uniquely determined by its $(-1,-1)$-cyclic displacement and any one of its rows or columns.

\section{Inversion of CUML Toeplitz and CUML Hankel matrices}

Before stating the main results below, we first introduce the related notations.

Theorem 4.1. Let $\mathrm{T}_{\mathrm{CUML}}$ be an arbitrary CUML Toeplitz matrix and let $\nabla_{-1,-1}(\cdot)$ be the linear operator in $\mathbf{C}^{\mathrm{n} \times \mathrm{n}}$ defined by (2.3). If the corresponding equations (3.2) and (3.3) have the solutions $\mathbf{f}_{\boldsymbol{i}}$ and $\hat{\mathbf{s}}_{\boldsymbol{i}}^{\top}(\boldsymbol{i}=1,2)$, respectively, then

$$
\sum_{i=1}^{2} \operatorname{RSFPLRcircfc}\left(\mathbf{f}_{i}\right) \cdot \operatorname{RSFPLRcircfr}\left(\mathbf{s}_{i}^{\top}\right)=0,
$$

where $\mathbf{s}_{i}^{\top}=\hat{\mathbf{s}}_{i}^{\top} \cdot \Xi_{-1,-1}^{-1}$.

Proof. As $\mathrm{T}_{\mathrm{CUML}}$ is an arbitrary $\mathrm{n} \times \mathrm{n}$ CUML Toeplitz matrix, then $\mathrm{T}_{\mathrm{CUML}}$ satisfies the condition

$$
\mathrm{T}_{\mathrm{CUML}}^{\mathrm{T}}=\mathrm{Z}_{-1} \mathrm{~J} \Xi_{-1,-1} \cdot \mathrm{T}_{\mathrm{CUML}} \cdot \Xi_{-1,-1}^{-1} \mathrm{JZ} \mathrm{Z}_{-1}^{-1}
$$

where

$$
Z_{-1}=\left(\begin{array}{ccccc}
0 & \cdots & \cdots & 0 & -1 \\
1 & 0 & & & 0 \\
0 & 1 & \ddots & & \vdots \\
\vdots & \ddots & \ddots & \ddots & \vdots \\
0 & \cdots & 0 & 1 & 0
\end{array}\right), \quad J=\left(\begin{array}{ccccc}
0 & \cdots & \cdots & 0 & 1 \\
\vdots & & . \cdot & 1 & 0 \\
\vdots & . \cdot & . \cdot & . \cdot & \vdots \\
0 & 1 & . \cdot & & \vdots \\
1 & 0 & \cdots & \cdots & 0
\end{array}\right),
$$

i.e., $Z_{-1}$ is the skew cyclic lower shift matrix [17], $J$ is the $n \times n$ reverse unit matrix, and $\Xi_{-1,-1}$ is the same as in the equation (2.3). Assume that $\Psi=Z_{-1} \mathrm{~J} \Xi_{-1,-1}$. Then

$$
\mathrm{T}_{\mathrm{CUML}}^{\mathrm{T}}=\Psi \mathrm{T}_{\mathrm{CUML}} \Psi^{-1} \text {, }
$$

where

$$
\Psi=\left(\begin{array}{ccccc}
0 & 0 & \ldots & 0 & 1 \\
0 & & . & 1 & -1 \\
\vdots & . \cdot & . \cdot & . \cdot & 0 \\
0 & 1 & . \cdot & . \cdot & \vdots \\
1 & -1 & 0 & \ldots & 0
\end{array}\right) \text { and } \Psi^{-1}=\left(\begin{array}{ccccc}
1 & 1 & \ldots & 1 & 1 \\
1 & & . \cdot & 1 & 0 \\
\vdots & . \cdot & . . & . \cdot & \vdots \\
1 & 1 & . \cdot & & 0 \\
1 & 0 & \ldots & 0 & 0
\end{array}\right)
$$

For $T_{C U M L}$ and the representation (3.1) with a given $\beta \in \mathbf{C}$ of its $(-1,-1)$-cyclic displacement, we assume that the corresponding equations (3.2) and (3.3) have the solutions $\mathbf{f}_{1}$ and $\mathbf{f}_{2}$, and $\hat{\mathbf{s}}_{1}^{\top}$ and $\hat{\mathbf{s}}_{2}^{\top}$, respectively, that is,

$$
\mathrm{T}_{\mathrm{CUML}} \mathbf{f}_{1}=\mathbf{x}, \mathrm{T}_{\mathrm{CUML}} \mathbf{f}_{2}=\mathbf{e}_{0}
$$


and

$$
\hat{\mathbf{s}}_{1}^{\top} \mathrm{T}_{\mathrm{CUML}}=\mathbf{e}_{0}^{\top} \cdot \Xi_{-1,-1,}, \hat{\mathbf{s}}_{2}^{\top} \mathrm{T}_{\mathrm{CUML}}=\mathbf{z}^{\top} \cdot \Xi_{-1,-1} .
$$

Set, as in equation (2.9),

$$
\mathbf{s}_{1}^{\top}=\hat{\mathbf{s}}_{1}^{\top} \cdot \Xi_{-1,-1}^{-1}, \mathbf{s}_{2}^{\top}=\hat{\mathbf{s}}_{2}^{\top} \cdot \Xi_{-1,-1}^{-1} \cdot
$$

Performing transformations to both equations in (4.3) and taking into account the identity (4.2), and $\mathbf{e}_{0}^{\top}=-\mathbf{e}_{0}^{\top} Z_{-1} J, \mathbf{z}^{\top}=\mathbf{x}^{\top} Z_{-1} J$, we can get that vectors $\mathbf{s}_{1}^{\top}$ and $\mathbf{s}_{2}^{\top}$ are related with the solutions $\mathbf{f}_{2}=$ $\left(f_{2,0}, f_{2,1}, \cdots, f_{2, n-1}\right)^{\top}$ and $\mathbf{f}_{1}=\left(f_{1,0}, f_{1,1}, \cdots, f_{1, n-1}\right)^{\top}$ of the equations $T_{C U M L} f_{2}=e_{0}$ and $T_{C U M L} f_{1}=\mathbf{x}$ in the following way

$$
\mathbf{s}_{1}^{\top}=\mathbf{e}_{0}^{\top} \cdot \Xi_{-1,-1} \cdot \mathrm{T}_{\mathrm{CUML}}^{-1} \Xi_{-1,-1}^{-1}=-\mathbf{f}_{2}^{\top} \mathrm{Z}_{-1} \mathrm{~J}, \quad \mathbf{s}_{2}^{\top}=\mathbf{z}^{\top} \cdot \Xi_{-1,-1} \cdot \mathrm{T}_{\mathrm{CUML}}^{-1} \Xi_{-1,-1}^{-1}=\mathbf{f}_{1}^{\top} \mathrm{Z}_{-1} \mathrm{~J} .
$$

These imply that $\mathbf{s}_{1}^{\top}=\left(f_{2,0}-f_{2, n-1} \cdots-f_{2,1}\right)$ is the first row of the matrix RSFPLRcircfc $\left(\mathbf{f}_{2}\right)$, and $-\mathbf{s}_{2}^{\top}=\left(f_{1,0}-f_{1, n-1} \cdots-f_{1,1}\right)$ is the first row of the matrix RSFPLRcircfc $\left(\mathbf{f}_{1}\right)$. Hence,

$$
\operatorname{RSFPLRcircfc}\left(\mathbf{f}_{1}\right)=-\operatorname{RSFPLR} \operatorname{circfr}\left(\mathbf{s}_{2}^{\top}\right), \quad \operatorname{RSFPLRcircfc}\left(\mathbf{f}_{2}\right)=\operatorname{RSFPLRcircfr}\left(\mathbf{s}_{1}^{\top}\right) .
$$

As

$$
\operatorname{RSFPLRcircfr}\left(\mathbf{f}_{1}\right) \operatorname{RSFPLR} \operatorname{circfr}\left(\mathbf{f}_{2}\right)=\operatorname{RSFPLR} \operatorname{circfr}\left(\mathbf{f}_{2}\right) \operatorname{RSFPLRcircfr}\left(\mathbf{f}_{1}\right),
$$

the assertion (ii) is now completely proved.

Next, we give the main results of this paper.

Theorem 4.2. Let $\mathrm{T}_{\mathrm{CUML}}$ be a CUML Toeplitz matrix with the $(-1,-1)$-cyclic displacement being given in (3.1) as the outer sum $\nabla_{-1,-1}\left(\mathrm{~T}_{\mathrm{CUML}}\right)=\mathbf{x} \cdot \mathbf{e}_{0}^{\mathrm{T}}+\mathbf{e}_{0} \cdot \mathbf{z}^{\mathrm{T}}$.

(i) If the equations (3.2), (3.3), and (2.10) have the corresponding solutions $\mathbf{f}_{i}, \hat{\mathbf{s}}_{i}^{\top}(i=1,2)$ and $\mathbf{y}_{1}^{\top}$, then $\mathrm{T}_{\mathrm{CUML}}$ is invertible and $\mathrm{T}_{\mathrm{CUML}}^{-1}$ is of the form as

$$
\mathrm{T}_{\text {CUML }}^{-1}=\operatorname{RSFPLRcircfr}\left(\mathbf{y}_{1}^{\top}\right)-\frac{1}{2} \sum_{i=1}^{2} \operatorname{RSFPLRcircfc}\left(\mathbf{f}_{i}\right) \cdot \operatorname{RFMLRcircfr}\left(\mathbf{s}_{i}^{\top}\right),
$$

where $\mathbf{s}_{i}^{\top}=\hat{\mathbf{s}}_{i}^{\top} \cdot \Xi_{-1,-1}^{-1}(\mathfrak{i}=1,2)$ and $\operatorname{RSFPLR} \operatorname{Rircfr}\left(\mathbf{y}_{1}^{\top}\right)$ is a row skew first-plus-last right circulant matrix with the first row $\mathbf{y}_{1}^{\top}$.

(ii) If the equations (3.2), (3.3), and (2.11) have the corresponding solutions $\mathbf{f}_{i}, \hat{\mathbf{s}}_{i}^{\top}(i=1,2)$ and $\mathbf{y}_{2}$, then $\mathrm{T}_{\mathrm{CUML}}$ is invertible and $\mathrm{T}_{\mathrm{CUML}}^{-1}$ may also be written in the form

$$
\mathrm{T}_{\text {CUML }}^{-1}=\operatorname{RSFPLRcircfc}\left(\mathbf{y}_{2}\right)-\frac{1}{2} \sum_{i=1}^{2} \operatorname{RFMLRcircfc}\left(\mathbf{f}_{i}\right) \cdot \operatorname{RSFPLRcircfr}\left(\mathbf{s}_{i}^{\top}\right),
$$

where $\mathbf{s}_{i}^{\top}=\hat{\mathbf{s}}_{i}^{\top} \cdot \Xi_{-1,-1}^{-1}(i=1,2)$ and $\operatorname{RSFPLR} \operatorname{circfc}\left(\mathbf{y}_{2}\right)$ is a row skew first-plus-last right circulant with the first column $\mathbf{y}_{2}$.

Proof. Due to Theorem 2.1, the solvability of the corresponding equations (3.2) yields the invertibility of $\mathrm{T}_{\mathrm{CUML}}$. Then the equations (3.3) are also solvable.

Now, we shall verify the equation (4.4). Observe Theorem 4.1 that vectors $\mathbf{f}_{i}, \mathbf{s}_{i}^{\top}=\hat{\mathbf{s}}_{i}^{\top} \cdot \Xi_{-1,-1}^{-1}(\mathfrak{i}=$ $1,2)$ satisfy the condition (4.1), where $\mathbf{f}_{i}, \hat{\mathbf{s}}_{i}^{\top}(\boldsymbol{i}=1,2)$ are the solutions of the equations (3.2) and (3.3), respectively, and calculate the $(-1,-1)$-cyclic displacement of the matrix on the right hand side of (4.4), denoted by $B$. The matrices RSFPLRcircfc $\left(\mathbf{f}_{\mathfrak{i}}\right)(i=1,2)$ are row skew first-plus-last right circulants, and 
$\operatorname{RFMLR\operatorname {circfr}}\left(\mathbf{s}_{i}^{\top}\right)(i=1,2)$ are row first-minus-last right circulants and hence are computable. From (4.4), we deduce that:

$$
\begin{aligned}
& \nabla_{-1,-1}(\mathrm{~B})=\nabla_{-1,-1}\left(\operatorname{RSFPLR} \operatorname{Rircfr}\left(\mathbf{y}_{1}^{\top}\right)\right)-\frac{1}{2} \sum_{\mathbf{i}=1}^{2} \nabla_{-1,-1}\left[\operatorname{RSFPLRcircfc}\left(\mathbf{f}_{\mathfrak{i}}\right) \cdot \operatorname{RFMLRcircfr}\left(\mathbf{s}_{\mathbf{i}}^{\top}\right)\right] \\
& =\nabla_{-1,-1}\left(\operatorname{RSFPLRcircfr}\left(\mathbf{y}_{1}^{\top}\right)\right)-\frac{1}{2} \sum_{i=1}^{2} \operatorname{RSFPLRcircfc}\left(\mathbf{f}_{i}\right) \cdot \nabla_{-1,-1}\left[\operatorname{RFMLRcircfr}\left(\mathbf{s}_{i}^{\top}\right)\right] .
\end{aligned}
$$

The last equality follows from (2.3) and Theorem 4.1, as

$$
\begin{aligned}
& \nabla_{-1,-1}\left[\operatorname{RSFPLR\operatorname {circfc}}\left(\mathbf{f}_{i}\right) \cdot \operatorname{RFMLR\operatorname {circfr}}\left(\mathbf{s}_{i}^{\top}\right)\right] \\
& =\operatorname{RSFPLRcircfc}\left(\mathbf{f}_{i}\right) \cdot \operatorname{RFMLRcircfr}\left(\mathbf{s}_{i}^{\top}\right) \\
& -\Xi_{-1,-1} \operatorname{RSFPLRcircfc}\left(\mathbf{f}_{i}\right) \Xi_{-1,-1}^{-1} \cdot \Xi_{-1,-1} \operatorname{RFMLRcircfr}\left(\mathbf{s}_{i}^{\top}\right) \Xi_{-1,-1}^{-1} \\
& =\operatorname{RSFPLRcircfc}\left(\mathbf{f}_{i}\right)\left[\operatorname{RFMLRcircfr}\left(\mathbf{s}_{i}^{\top}\right)-\Xi_{-1,-1} \operatorname{RFMLRcircfr}\left(\mathbf{s}_{i}^{\top}\right) \Xi_{-1,-1}^{-1}\right] \\
& =\operatorname{RSFPLR} \operatorname{circfc}\left(\mathbf{f}_{i}\right) \cdot \nabla_{-1,-1}\left[\operatorname{RFMLR} \operatorname{circfr}\left(\mathbf{s}_{i}^{\top}\right)\right], \quad i=1,2 \text {. }
\end{aligned}
$$

Computing in this way the $(-1,-1)$-cyclic displacement for each matrix $\operatorname{RFMLRcircfr}\left(\mathbf{s}_{i}^{\top}\right)$ on the right hand side of (4.6) and taking into account the fact that $\nabla_{-1,-1}\left(\operatorname{RSFPLRcircfr}\left(\mathbf{y}_{1}^{\top}\right)\right)=0$ (see (i) of Theorem $3.2)$, we obtain

$$
\nabla_{-1,-1}(\mathrm{~B})=\sum_{i=1}^{2} \operatorname{RSFPLRcircfc}\left(\mathbf{f}_{i}\right) \cdot \tilde{\mathbf{s}}_{i} \cdot \mathbf{e}_{0}^{\top}-\sum_{i=1}^{2} \operatorname{RSFPLRcircfc}\left(\mathbf{f}_{i}\right) \cdot \mathbf{e}_{0} \cdot \mathbf{s}_{i}^{\top},
$$

where $\tilde{\mathbf{s}}_{i}$ is the first column of the RSFPLRcircfr $\left(\mathbf{s}_{i}^{\top}\right)(i=1,2)$. Therefore, in view of Theorem 4.1, the first two terms on the right of (4.7) are equal to the zero matrix. Furthermore, RSFPLRcircfc $\left(\mathbf{f}_{i}\right) \cdot \mathbf{e}_{0}=\mathbf{f}_{i}$ $(i=1,2)$, and hence the matrix B satisfies (4.6), $\nabla_{-1,-1}(B)=-\sum_{i=1}^{2} \mathbf{f}_{i} \cdot \mathbf{s}_{i}^{\top}$, so that by (3.4), $\nabla_{-1,-1}\left(\mathrm{~T}_{\mathrm{CUML}}^{-1}\right)=$ $\nabla_{-1,-1}(\mathrm{~B})$, therefore, in view of (4.1), the first rows of the matrices $\mathrm{T}_{\text {CUML }}^{-1}$ and B (or RSFPLRcircfr $\left(\mathbf{y}_{1}^{\top}\right)$ ) coincide. Thus $\mathrm{B}=\mathrm{T}_{\text {CUML }}^{-1}$. The proof of assertion (i) is now completed.

The assertion (ii) can be proved in the same way as that given in (i).

From (i) and (ii) of Theorem 4.2, we further deduce the following.

Theorem 4.3. Let $\mathrm{T}_{\mathrm{CUML}}$ be a CUML Toeplitz matrix and let $\nabla_{-1,-1}\left(\mathrm{~T}_{\mathrm{CUML}}\right)$ be of the form as in (3.1). If $\beta \in \mathbf{C}$ and the equations (3.2) have the solutions $\mathbf{f}_{1}$ and $\mathbf{f}_{2}$, then $\mathrm{T}_{\mathrm{CUML}}$ is invertible and $\mathrm{T}_{\mathrm{CUML}}^{-1}$ is of the form as

$$
\mathrm{T}_{\mathrm{CUML}}^{-1}=\frac{1}{2}\left[\operatorname{RFMLRcircfc}\left(2 \mathbf{e}_{0}-\mathbf{f}_{1}\right) \cdot \operatorname{RSFPLRcircfc}\left(\mathbf{f}_{2}\right)+\operatorname{RFMLRcircfc}\left(\mathbf{f}_{2}\right) \cdot \operatorname{RSFPLRcircfc}\left(\mathbf{f}_{1}\right)\right] .
$$

Proof. In view of (i) of Theorem 4.2, $\mathrm{T}_{\text {CUML }}^{-1}$ does exist under the assumption of the theorem. We only need to show that the formula (4.8) holds. Let the vectors $\mathbf{f}_{1}$ and $\mathbf{f}_{2}$ be the solutions of the equations (3.2). By $\mathrm{T}_{\mathrm{CUML}} \mathbf{f}_{2}=\mathbf{e}_{0}$, we have RSFPLRcircfc $\left(\mathrm{T}_{\mathrm{CUML}}^{-1}\right)=\operatorname{RSFPLRcircfc}\left(\mathbf{f}_{2}\right)$; furthermore, in view of the arguments of Theorem 4.1, RSFPLRcircfc $\left(\mathbf{f}_{1}\right)=-\operatorname{RSFPLRcircfr}\left(\mathbf{s}_{2}^{\top}\right)$, and RSFPLRcircfc $\left(\mathbf{f}_{2}\right)=\operatorname{RSFPLRcircfr}\left(\mathbf{s}_{1}^{\top}\right)$. Now, wielding these expressions and (4.5), we can get the desired result.

Theorem 4.3 states that if $\mathrm{T}_{\mathrm{CUML}}$ is a CUML Toeplitz matrix and the equations (3.2) have solutions $\mathbf{f}_{1}$ and $\mathbf{f}_{2}$, then they are sufficient for restoring the whole matrix $T_{C U M L}^{-1}$.

Another representation of the inverse of a CUML Toeplitz matrix is obtained in the following theorem. 
Theorem 4.4. Let $\mathrm{T}_{\mathrm{CUML}}$ be a CUML Toeplitz matrix of the form as in (2.1). If for some $\gamma \in \mathbf{C}$ and the equations

$$
\mathrm{T}_{\mathrm{CUML}} \mathbf{f}_{2}=\mathbf{e}_{0} \text { and } \mathrm{T}_{\mathrm{CUML}} \mathbf{s}=\left(\gamma, \mathrm{t}_{-\mathrm{n}+1}, \cdots, \mathrm{t}_{-1}\right)^{\mathrm{T}}
$$

are solvable, then $\mathrm{T}_{\mathrm{CUML}}$ is invertible, and $\mathrm{T}_{\mathrm{CUML}}^{-1}$ is of the form as

$$
\mathrm{T}_{\mathrm{CUML}}^{-1}=\frac{1}{2}\left[\operatorname{RFMLR\operatorname {Rirfc}}\left(\mathbf{e}_{0}-\mathbf{s}\right) \operatorname{RSFPLR} \operatorname{Rircfc}\left(\mathbf{f}_{2}\right)+\operatorname{RFMLR\operatorname {Rircfc}}\left(\mathbf{f}_{2}\right) \operatorname{RSFPLRcircfc}\left(\mathbf{e}_{0}+\mathbf{s}\right)\right] .
$$

Proof. Let $\mathbf{s} \in \mathbf{C}^{n}$ be a solution of the second equation in (4.9). Then the vector $\mathbf{f}_{1}=\mathbf{e}_{0}+\mathbf{s}$ solves the first equation in (3.2) with $\beta=t_{0}+\gamma$, that is, $T_{\text {CUML }}\left(\mathbf{e}_{0}+\mathbf{s}\right)=\left(t_{0}+\gamma, t_{1}+t_{1-n}, \cdots, t_{n-1}+t_{-1}\right)^{\top}$. Thus the results of Theorem 4.4 are straightforward consequence of Theorem 4.3.

According to the definitions and characteristics of CUML Toeplitz matrices and CUML Hankel matrices, the relationship between them is easy to comprehend. Thus, we can obtain the inverses of CUML Hankel matrices.

Theorem 4.5. Let $\mathrm{H}_{\mathrm{CUML}}=\left(\mathrm{h}_{\mathrm{i}, \mathrm{j}}\right)_{i, j=0}^{\mathrm{n}-1}$ be an arbitrary CUML Hankel matrix defined by (2.2), and let $\mathrm{J}$ be the reverse unit matrix. Then an $\mathrm{n} \times \mathrm{n}$ CUML Toeplitz matrix $\mathrm{T}_{\mathrm{CUML}}$ exists which satisfies that $\mathrm{T}_{\mathrm{CUML}}=\mathrm{H}_{\mathrm{CUML}} \mathrm{J}$, and $\mathrm{H}_{\mathrm{CUML}}$ is invertible if and only if $\mathrm{T}_{\mathrm{CUML}}$ is also. In that case the inverse of matrix $\mathrm{H}_{\mathrm{CUML}}$ is $\mathrm{H}_{\mathrm{CUML}}^{-1}=\mathrm{JT}_{\mathrm{CUML}}^{-1}$ and Theorems 4.2, 4.3, and 4.4 are applicable to describe the formula on representation of the inverse of $\mathrm{H}_{\mathrm{CUML}}$.

\section{Acknowledgment}

This work was supported by the Natural Science Foundation of China under Grant No. 11271175. Their support is gratefully acknowledged.

\section{References}

[1] G. Ammar, P. Gader, New decompositions of the inverse of a Toeplitz matrix, Signal processing, scattering and operator theory, and numerical methods, Amsterdam, (1989), Progr. Systems Control Theory, Birkhäuser Boston, Boston, MA, 5 (1990), 421-428. 1, 3

[2] G. Ammar, P. Gader, A variant of the Gohberg-Semencul formula involving circulant matrices, SIAM J. Matrix Anal. Appl., 12 (1991), 534-540. 1

[3] Y.-Q. Bai, T.-Z. Huang, X.-M. Gu, Circulant preconditioned iterations for fractional diffusion equations based on Hermitian and skew-Hermitian splittings, Appl. Math. Lett., 48 (2015), 14-22. 1

[4] A. Ben-Artzi, T. Shalom, On inversion of Toeplitz and close to Toeplitz matrices, Linear Algebra Appl., 75 (1986), 173-192. 1

[5] A. Brown, P. R. Halmos, Algebraic properties of Toeplitz operators, J. Reine Angew. Math., 213 (1964), 89-102. 1

[6] C. C. Cowen, Hyponormality of Toeplitz operators, Proc. Amer. Math. Soc., 103 (1988), 809-812.

[7] D. R. Farenick, W. Y. Lee, Hyponormality and spectra of Toeplitz operators, Trans. Amer. Math. Soc., 348 (1996), 4153-4174. 1

[8] P. D. Gader, Displacement operator based decompositions of matrices using circulants or other group matrices, Linear Algebra Appl., 139 (1990), 111-131. 1, 3

[9] I. Gohberg, V. Olshevsky, Circulants, displacements and decompositions of matrices, Integral Equations Operator Theory, 15 (1992), 730-743. 1, 1

[10] G. Heinig, On the reconstruction of Toeplitz matrix inverses from columns, Linear Algebra Appl., 350 (2002), $199-212$. 1

[11] I. S. Hwang, W. Y. Lee, Block Toeplitz operators with rational symbols, J. Phys. A, 41 (2008), 7 pages. 1

[12] Z.-L. Jiang, J.-X. Chen, The explicit inverse of nonsingular conjugate-Toeplitz and conjugate-Hankel matrices, J. Appl. Math. Comput., 53 (2017), 1-16. 1

[13] Z.-L. Jiang, X.-T. Chen, J.-M. Wang, The explicit inverses of CUPL-Toeplitz and CUPL-Hankel matrices, East Asian J. Appl. Math., 7 (2017), 38-54. 1

[14] X.-Y. Jiang, K.-C. Hong, Explicit determinants of the k-Fibonacci and k-Lucas RSFPLR circulant matrix in codes, Inf. Comput. Appl., Springer, Berlin, Heidelberg, (2013), 625-637. 3

[15] X.-Y. Jiang, K.-C. Hong, Algorithms for finding inverse of two patterned matrices over $\mathbb{Z}_{\mathrm{p}}$, Abstr. Appl. Anal., 2014 (2014), 6 pages. 
[16] X.-Y. Jiang, K.-C. Hong, Exact determinants of some special circulant matrices involving four kinds of famous numbers, Abstr. Appl. Anal., 2014 (2014), 12 pages. 3

[17] X.-Y. Jiang, K.-C. Hong, Explicit inverse matrices of Tribonacci skew circulant type matrices, Appl. Math. Comput., 268 (2015), 93-102. 3.1, 4

[18] X.-Y. Jiang, K.-C. Hong, Skew cyclic displacements and inversions of two innovative patterned matrices, Appl. Math. Comput., 308 (2017), 174-184. 1

[19] Z. L. Jiang, Y. C. Qiao, S. D. Wang, Norm equalities and inequalities for three circulant operator matrices, Acta Math. Sin. (Engl. Ser.), 33 (2017), 571-590. 3.1

[20] Z.-L. Jiang, T.-Y. Tam, Y.-F. Wang, Inversion of conjugate-Toeplitz matrices and conjugate-Hankel matrices, Linear Multilinear Algebra, 65 (2017), 256-268. 1

[21] Z.-L. Jiang, D.-D. Wang, Explicit group inverse of an innovative patterned matrix, Appl. Math. Comput., 274 (2016), 220-228. 1

[22] Z.-L. Jiang, T.-T. Xu, Norm estimates of $\omega$-circulant operator matrices and isomorphic operators for $\omega$-circulant algebra, Sci. China Math., 59 (2016), 351-366. 3.1

[23] T. Kailath, S. Y. Kung, M. Morf, Displacement ranks of matrices and linear equations, J. Math. Anal. Appl., 68 (1979), 395-407. 1

[24] S.-L. Kong, Z.-S. Zhang, Optimal control of stochastic system with Markovian jumping and multiplicative noises, Acta Automat. Sinica, 38 (2017), 1113-1118. 1

[25] G. Labahn, T. Shalom, Inversion of Toeplitz matrices with only two standard equations, Linear Algebra Appl., 175 (1992), 143-158. 1

[26] G. Labahn, T. Shalom, Inversion of Toeplitz structured matrices using only standard equations , Linear Algebra Appl., 207 (1994), 47-70. 1

[27] L. Lerer, M. Tismenetsky, Generalized Bezoutian and the inversion problem for block matrices, I, General scheme, Integral Equations Operator Theory, 9 (1986), 790-819. 1

[28] X.-G. Lv, T.-Z. Huang, A note on inversion of Toeplitz matrices, Appl. Math. Lett., 20 (2007), 1189-1193. 1

[29] M. K. Ng, J.-Y. Pan, Weighted Toeplitz regularized least squares computation for image restoration, SIAM J. Sci. Comput., 36 (2014), B94-B121. 1

[30] M. K. Ng, K. Rost, Y.-W. Wen, On inversion of Toeplitz matrices, Linear Algebra Appl., 348 (2002), 145-151. 1

[31] D. Potts, G. Steidl, Preconditioners for ill-conditioned Toeplitz matrices, BIT, 39 (1999), 513-533. 1

[32] N. Shen, Z.-L. Jiang, J. Li, On explicit determinants of the RFMLR and RLMFL circulant matrices involving certain famous numbers, WSEAS Trans. Math., 12 (2013), 42-53. 2, 2, 3

[33] Y.-P. Zheng, S.-G. Shon, Exact determinants and inverses of generalized Lucas skew circulant type matrices, Appl. Math. Comput., 270 (2015), 105-113. 3.1

[34] Y.-P. Zheng, S.-G. Shon, J.-Y. Kim, Cyclic displacements and decompositions of inverse matrices for CUPL Toeplitz matrices, J. Math. Anal. Appl., 455 (2017), 727-741. 1 